\title{
Autoconcepto, Locus de Control y ORIentación al Éxito: sus Relaciones Predictivas en Adultos Mayores del Noreste Brasileño
}

\section{José Angel Vera Noriega1, Miriam Teresa Dominguez Guedea ${ }^{2}$, Jesús Francisco Laborín Álvarez ${ }^{3}$, Francisco José Batista Albuquerque ${ }^{4}$ e Magno Seabra ${ }^{5}$}

Centro de investigación en Alimentación y Desarrollo

Universidad de Sonora

Universidade Federal da Paraiba

\begin{abstract}
El objetivo fue analizar las relaciones predictivas entre el autoconcepto, locus de control, orientación al éxito y evitación al fracaso en una muestra de adultos mayores de João Pessoa, Brasil. Participaron en este estudio 123 personas adultos mayores con edades entre 60 y 93 años, de las cuales, la mitad era del sexo masculino y la otra mitad de sexo femenino. Se utilizaron escalas de locus de control, autoconcepto y orientación al éxito validadas en población pessoense, así como un cuestionario sociodemográfico diseñado exprofesso. Se utilizaron análisis de regresión jerárquica, encontrando diversas relaciones predictivas entre aspectos de personalidad, confirmando el poder explicativo de las variables locus de control interno y externo, autoconcepto positivo y negativo y evitación al fracaso.
\end{abstract}

Descriptores: Local de control interno-externo. Autoimagen. Adultos mayores.

1 Centro de investigación en Alimentación y Desarrollo, A.C. , Hermosillo, Sonora, México. Endereço eletrônico: avera@ cascabel.ciad.mx

2 Universidad de Sonora, Hermosillo Sonora, México. Endereço eletrônico: teresad@ sociales.uson.mx

3 Centro de investigación en Alimentación y Desarrollo, A.C. Endereço eletrônico: laborin@ciad.mx

4 Universidade Federal de Paraiba, João Pessoa, Brasil. Endereço eletrônico: frajoba@ uol.com.br

5 Universidade Federal de Paraiba, João Pessoa, Brasil. Endereço eletrônico: magnoalexon@hotmail.com 


\begin{abstract}
partir de 1960, en Brasil se han venido registrando aumentos constantes en el número de personas con 60 o más años. De acuerdo a las tendencias demográficas registradas por el Instituto Nacional de Geografía y Estadística de Brasil (IBGE, 2006), el índice de envejecimiento en el territorio nacional en el año 2000 fue de 19,77\%, cifra que representó casi el doble de ese mismo indicador en relación a las dos décadas anteriores. Estas proporciones tienden al aumento, tanto que la Organización Mundial de la Salud ha proyectado que para el año 2025, Brasil ocupará el sexto lugar en el mundo con la mayor cantidad de personas en la fase de la tercera edad (Ministério da Saúde, 2006).
\end{abstract}

Existe una dicotomía en lo que se refiere a la jubilación, dado que puede ser asociada a la interrupción de la actividad laboral, percibida como un periodo de la vida que va en decline, conduciendo a las personas a que se consideren inútiles, desvalorizados y sin motivaciones. Y por otro lado, la jubilación puede ser entendida también como un estado de reposo, un derecho adquirido y conquistado por la ciudadanía.

En su propuesta teórica sobre el desarrollo del ciclo vital, Erikson (1968, citado en Carver \& Scheier, 1994, p. 313) describe con detalle la etapa de madurez o vejez y la entiende como el capítulo final de la vida, como un tiempo en el que la gente mira hacia atrás para revisar sus elecciones, en el que la persona reflexiona sobre sus logros (y fracasos) y sobre el rumbo que tomó su vida. Siguiendo al autor, esta etapa está caracterizada, por la crisis "integridad del yo frente a la desesperación"; si en el curso de esta revisión personal el individuo siente que su vida tuvo orden y significado, hubo la aceptación de sus decisiones y de las cosas realizadas, el resultado será un sentimiento de integridad del yo, de una satisfacción que supone que las cosas sucedieron como se deseaba y que no se cambiaría mucho de ellas, aunque fuera posible. El polo opuesto es la desesperación, el sentimiento de que la vida fue desperdiciada, un deseo de haber hecho las cosas de manera diferente a sabiendas de que es demasiado tarde. En lugar de aceptar la historia que constituye su vida como un presente valioso, el individuo siente amargura por la forma en que resultaron las cosas

Durante la tercera edad tienen lugar una serie de cambios neurobiológicos, físicos, psicológicos, familiares y sociales que demandan del adulto mayor, recursos personales que le ayuden a logar la adaptación en esta etapa de la vida 
Autoconcepto, Locus de Control y Orientación al Éxito: sus Relaciones Predictivas...

(Domínguez-Guedea, 2002). Sobre estos recursos, existen investigaciones que apuntan a la percepción de bienestar y apoyo social, las estrategias utilizadas para afrontar los problemas, la personalidad, la autoestima y la capacidad de control como aspectos importantes para un envejecimiento exitoso o saludable (Cava \& Musitu, 2000; Gayoso, 2004; Hombrados \& García, 2002).

Este trabajo se centró en el análisis de la relación que guardan entre sí, tres aspectos considerados componentes de la personalidad: autoconcepto, locus de control y orientación al éxito. El locus de control interno, es un aspecto que puede ayudar a los adultos mayor en el auto-cuidado de su salud pues ha demostrado relacionarse con la adherencia terapéutica entre pacientes geriátricos con diabetes mellitus (Díaz, Galán, \& Fernández, 1993) y además es un componente importante del empoderamiento, como estrategia para preservar la salud propia (Texeira, 2002); por su parte, el locus de control externo, puede ser visto como un factor de vulnerabilidad que predispone al padecimiento de trastornos de ansiedad en la tercera edad (Francés, 2004). El autoconcepto positivo se relaciona con la disposición del adulto mayor a realizar actividades físicas, que a su vez favorece la autonomía funcional (Mora, Villalobos, Araya, \& Ozols, 2004). Por otro lado, la falta de motivación acerca de objetivo en la vida ha sido indicado como un factor de riesgo que favorece el deterioro cognitivo en el adulto mayor (OMS, 2001; Puig, 2000). A continuación, se detallan algunas consideraciones conceptuales acerca de estos tres constructos.

La literatura indica que el locus de control es un aspecto de la personalidad que se relaciona con la forma en la cual un adulto mayor enfrenta los eventos estresantes, de tal manera que los adultos mayores que poseen control interno con frecuencia enfrentan o resuelven sus problemas empleando conductas adaptativas. Quien tiene una fuerte creencia en el control interno es más probable que vea las situaciones estresantes como controlables y busque alternativas de solución. De otra forma las personas controladas externamente podrían encontrar que la solución a los problemas depende de la suerte, del destino o dios (Peacock \& Wong, 1996); Sin embargo los estudios llevados a cabo por Vera-Noriega y Cervantes (2000) se concluye que cuanto mayor sea la edad, menor será el locus de control interno. Esto fue encontrado con una muestra que contaba con personas de edades a partir de los catorce hasta los setenta años residentes del estado de Sonora México. 
El autoconcepto desempeña un papel importante en el bienestar subjetivo (Herzog, Franks, Markus, \& Holmberg, 1998), a lo largo de todos los ciclos de vida. El reconocimiento del papel del autoconcepto en el bienestar personal no es reciente, pues ya desde las civilizaciones griegas, hindú, budista, judeocristiana, hasta nuestros días se ha indicado la relevancia que posee el autoconcepto como factor que determina la organización del propio comportamiento, como vía de crecimiento y de integridad personal (DomínguezGuedea, 1999). En lo que se refiere a la tercera edad, Corral y Ortiz (1990) mencionan que ante las pérdidas biológicas, psicológicas y cambios sociales vividos por los adultos mayores, su autoconcepto puede verse alterado, siendo importante analizar la percepción de sí mismo durante esta etapa de la vida y observar su relación con el bienestar en la tercera edad.

Martínez y García (1994), relacionan la motivación al éxito con el bienestar psicológico que, como constructo multidimensional está ligado al sistema de valores, aspiraciones, expectativas y metas de los individuos, consideran que la percepción de satisfacción con la vida esta condicionada por la historia personal de éxito. En concordancia con esto Emmons (1986), señala que en la cultura occidental existe la creencia de tener que alcanzar metas en la vida como una regla de bienestar para las personas, así que las satisfacciones de las necesidades de las metas o deseos están relacionados con la felicidad.

En relación al factor de personalidad, autoconcepto y locus de control, como predictores, se considera que el autoconcepto influye en la evolución cognitiva de la situación estresante facilitando un comportamiento activo centrado en un problema y la consecución de resultados positivos. Acerca del locus de control las investigaciones indican que las personas con locus de control interno muestran más persistencia y se involucran en la obtención de buenos resultados ante las crisis eventuales buscando información y presentando más conductas preventivas en relación a la problemática (Hudek \& Kardum, 1996).

Este estudio tuvo como objetivo analizar las relaciones predictivas entre el autoconcepto, locus de control, orientación al éxito y evitación al fracaso en una muestra de adultos mayores de João Pessoa, Brasil. 
Autoconcepto, Locus de Control y Orientación al Éxito: sus Relaciones Predictivas...

\section{Procedimientos}

\section{Participantes}

Se trabajó con una muestra no probabilística compuesta por 123 personas con edades de 60 hasta los 93 años (M=67; D.E=6). De los participantes, $49 \%$ eran mujeres, 45,5\% tenía estudios de secundaria o menos y el restante, estudios posteriores a ese grado de escolaridad; $58 \%$ vivía con una pareja; $60 \%$ era jubilado y $51 \%$ desarrollaba un trabajo con remuneración económica. Los criterios de inclusión a la muestra fueron: a) tener una residencia en João Pessoa mayor a los 20 años, con el objeto de asegurar que el adulto mayor adquirió las costumbres, las normas y reglas, vinculadas a ese contexto cultural; b) hablar y leer de manera fluida el portugués a fin de responder a los cuestionarios que se les fueron aplicados y; c) tener un ingreso familiar mensual de 800 reales a 1500 reales.

\section{Instrumentos de medida.}

Las escalas utilizadas cuentan con estudios de validación en población pessoense, cuyos resultados están reportados por Vera-Noriega, Alburquerque y Laborín (1999), Vera-Noriega, Alburquerque, Laborín, Morales y Torres (2002), Vera-Noriega, Alburquerque, Laborín, Souza y Coronado (2003) y Laborín-Álvarez y Vera (2000). A pesar de la validación de los instrumentos, análisis preliminares de los datos obtenidos en la muestra de adultos mayores identificaron una gran cantidad de ítems con distribuciones acentuadamente anormales. Se optó por utilizar sólo los ítems que no presentaron sesgo significativo de acuerdo al test de la asimetría, teniendo como criterio de eliminación de ítems aquellos con valores $\geq 3,29, p \leq 0,001$. Posteriormente se realizaron análisis factoriales y de Alpha de Cronbach para verificar las propiedades psicométricas de las medidas reducidas. Enseguida se describen brevemente los resultados así como el contenido de los instrumentos.

Escala de locus de control. Consta de 16 ítems que abordan las atribuciones de las personas acerca de los éxitos y fracasos en la vida y aspectos específicos tales como las relaciones interpersonales, condiciones económicas y de trabajo. Las opciones de respuesta están en escalas tipo Likert de cinco puntos ( $1=$ en desacuerdo; $5=$ en acuerdo). Se obtuvieron análisis factoriales utilizando 
el método de los ejes principales y rotación varimax, encontrando que todos los ítems alcanzaron saturaciones factoriales $\geq 0,33$. Los tres factores encontrados en la muestra de adultos explican el $38 \%$ de la varianza del constructo siendo denominados: 1) locus de control interno (cuatro ítems; alpha=0,76); 2) locus de control externo (seis ítems; alpha $=0,65$ ) y; 3 ) atribución interna-externa de éxito en la vida y las relaciones interpersonales (seis ítems; alpha=0,68).

Escala de autoconcepto. Con 17 ítems tipo Likert, la escala mide la forma en la cual una persona se describe a si misma, empleando adjetivos calificativos, en los que la proximidad de la respuesta a la palabra estímulo indica que se tiene mayor cantidad de ese atributo. Análisis factoriales (con el método de los ejes principales y rotación varimax) indicaron la presencia de dos factores en la muestra consultada, explicando el 39\% de la varianza y todos los ítems obtuvieron cargas factoriales $\geq 0,39$. Los factores fueron: 1 ) autoconcepto negativo interno (diez ítems; alpha $=0,77$ ) y; 2) autoconcepto positivo (siete ítems; alpha $=0,75$ ).

Escala de orientación y evitación al éxito. Se constituye por 30 enunciados referidos al éxito en el trabajo, la escuela, las relaciones de pareja, los logros personales, la familia y los amigos. Las opciones de respuesta están dispuestas en formato Likert de cinco puntos (1=nunca; 5=siempre). Análisis factoriales (con el método de los ejes principales y rotación varimax) indicaron la existencia de dos factores que explican $36 \%$ de la varianza del constructo: 1 ) factor evitación al fracaso (18 ítems, alpha $=0,87$ ) que es la tendencia a evitar las consecuencias afectivas negativas de fracasar y no conseguir el éxito (Horner, 1969) y ; 2) factor orientación al éxito (12 ítems, alpha=0,87) que trata del grado en el cual la persona se propone objetivos altos tratando de obtenerlos (McClelland, Atkinson, Clark, \& Lowell, 1961). Todos los ítems alcanzaron pesos factoriales $\geq 0,36$.

Además de estas 3 variables se aplicó un cuestionario de datos de identificación con el objeto de obtener un mapa de la situación del adulto mayor en relación a la pensión, jubilación a la actividad profesional, recursos económicos de la familia y la composición familiar. También se aplicó un formulario para identificar si el participante era alfabeto funcional (Moreira, 2003), esto, si era capaz, no sólo de leer, sino también de comprender y seguir instrucciones para el llenado de los instrumentos de medida. 
Autoconcepto, Locus de Control y Orientación al Éxito: sus Relaciones Predictivas...

Los instrumentos fueron aplicados en tres sesiones. Iniciando con los datos socio-demográficos y la identificación de la capacidad de lectura funcional del participante. En una segunda sesión se aplicaba la escala de locus de control y la de autoconcepto. En una tercera sesión se aplicaba la escala de orientación y de evitación al éxito.

\section{Resultados}

Previo a la revisión de las relaciones predictivas entre variables, se realizaron análisis para verificar la adecuación de los datos a los presupuestos multivariados de los tests estadísticos. Se analizaron las puntuaciones factoriales de: autoconcepto positivo, autoconcepto negativo, orientación al éxito, evitación al fracaso, locus de control interno, locus de control externo y atribución interna-externa de éxito en la vida y las relaciones interpersonales. Ninguna de las variables presentó anormalidad en sus distribuciones ni problemas de multicolinearidad (todos los valores de tolerancia fueron $\leq 0,65$ ); sin embargo, scatterplots entre las siete variables indicaron de manera inicial que no existen relaciones perfectamente lineales entre las puntuaciones factoriales. Se identificó un caso extremo multivariado (outlier multivariado) de acuerdo con la distancia de Mahalanobis de $\chi^{2}[(12)=26,22 ; \mathrm{p} \leq 0,01]$; se optó por retirar ese caso de los análisis posteriores.

En seguida se hicieron análisis de regresión jerárquica para determinar la capacidad predictiva de las variables de personalidad, una vez que la varianza de los indicadores socio-demográficos hubiera sido controlada. Para eso, el conjunto de predictoras fue agrupada en dos bloques: 1) variables socio-demográficas, teniendo a las variables $d u m m y^{6}$ : sexo femenino, vivir con la pareja, trabajar actualmente, vivir con nietos y contar con estudios superiores a la secundaria y; 2) variables de personalidad: autoconcepto, orientación/evitación al éxito y locus de control.

Platicaremos sobre los resultados de la regresión de un primer bloque de variables socio- demográficas teniendo a las puntuaciones factoriales de autoconcepto como variables criterio. Las variables socio-demográficas pre-

6 Código utilizado cuando una variable ordinal se transforma a dicotómica aplicando la fórmula K-1. Ver mas detalles en Tabachnick y Fidell (2001). 
sentaron una capacidad predictiva significativa sobre el autoconcepto positivo, $\left[R^{2}=0,09 ; p \leq 0,05\right]$. En seguida, el bloque de las variables de personalidad mejoraron notablemente el poder de predicción sobre el autoconcepto positivo $\left[R^{2}=0,28 ; p \leq 0,001\right]$. El modelo final contó con dos predictores positivos - trabajar actualmente y locus de control interno - y un predictor negativo - autoconcepto negativo -, indicando que las personas que económicamente activas y con una atribución interna acerca de los acontecimientos de la vida tienen un autoconcepto más positivo, así como aquellas que se describen a sí mismas en términos menos negativos. En relación a los resultados de autoconcepto negativo, el bloque de indicadores socio-demográficos no aumentó significativamente el valor de $R^{2}$, pero sí lo hicieron las variables de personalidad $\left[R^{2}=0,23 ; p \leq 0,001\right]$. Las dos variables predictoras fueron: autoconcepto positivo - con un coeficiente estandarizado negativo - y evitación al fracaso - con un coeficiente estandarizado positivo -. Lo anterior indica que aquellos que perciben menos características positivas en sí mismos y mayor temor a los fracasos, tienen un autoconcepto más negativo.

Un segundo conjunto de datos mostró los resultados relativos a las puntuaciones factoriales de orientación/evitación al éxito. Las variables sociodemográficas no presentaron predicción significativa sobre la orientación al éxito, pero las variables de personalidad sí lo hicieron $\left[R^{2}=0,25 ; p \leq 0,001\right]$. El modelo final mostró a evitación al fracaso y locus de control interno como predictores positivos, es decir, las personas que tienen mayor preocupación ante los fracasos y atribuyen a sí mismas lo que les sucede en la vida, están más orientados al éxito. Por otro lado, la VD "evitación al fracaso" contó con el 33\% de varianza explicada por los elementos de personalidad $\left[R^{2}=0,33 ; p \leq\right.$ 0,001], aún y cuando uno de sus predictores fue de orden socio-demográfico - trabajar actualmente -; los predictores de personalidad fueron autoconcepto negativo, orientación al éxito y locus de control externo. Esos resultados sugieren que los adultos mayores más preocupados con el fracaso son aquellos que trabajan actualmente, que tienen un concepto negativo de sí mismos y una atribución externa sobre la causalidad de los acontecimientos de la vida.

En un tercer plano hablaremos de los resultados para locus de control. El locus de control interno tuvo predictores socio-demográficos - sexo femenino y vivir con nietos $-\mathrm{y}$ de personalidad - autoconcepto positivo y orientación al éxito -. El modelo final obtuvo una $R^{2}=0,27 ; p \leq 0,001$, indicando que 
Autoconcepto, Locus de Control y Orientación al Éxito: sus Relaciones Predictivas...

las mujeres, quienes viven con sus nietos, tienen un concepto más positivo de sí mismo y están orientados al éxito, tienden atribuir a sí mismos los acontecimientos de la vida. En relación al locus de control externo, se encontró que el bloque de variables socio-demográficas tuvo un poder de explicación significativo $\left[R^{2}=0,11 ; p \leq 0,05\right]$, el cual se vio incrementado por las variables de personalidad $\left[R^{2}=0,46 ; p \leq 0,001\right]$; los predictores para este modelo apuntan que: mujeres, quienes tienen un autoconcepto negativo y una atribución interna y externa acerca del curso de las relaciones interpersonales y los éxitos obtenidos en la vida, tienen a tener un locus de control más externo. Finalmente, el único predictor significativo para la atribución interna-externa de éxito en las vida y las relaciones interpersonales fue locus de control externo, siendo que el modelo completo obtuvo una $R^{2}=0,32 ; p \leq 0,001$; lo anterior indica que las personas con que tienden a atribuir a causas externas los logros alcanzados en sus vida, tienden a tener una combinación interna y externa en la atribución de los resultados en sus relaciones interpersonales.

\section{Discusión}

Ante el inminente aumento de los índices de envejecimiento a nivel mundial, se han movilizado múltiples esfuerzos para identificar los factores que pueden ayudar a atenuar los problemas de salud típicos de la tercera edad (Domínguez-Guedea, 2002). De esta preocupación se ha desprendido un cambio en la conceptualización de la vejez, de acuerdo a la cual, es necesario prestar atención a los recursos personales y sociales que permitan fomentar la salud y el autocuidado, y no sólo centrarse en el tratamiento de las enfermedades. Ya desde 1982, la Organización Panamericana de la Salud definía a la salud del adulto mayor en términos de la capacidad funcional versus la tradicional perspectiva de déficit. De esta manera, las políticas internacionales están orientadas al mantenimiento de la funcionalidad entre los adultos mayores, para lo cual, es necesario detectar en forma anticipada aquellos factores que la promueven o bien, la dificultan (Sanhueza, Castro, \& Merino, 2005).

Los resultados de esta investigación hacen una contribución al conjunto de estudio interesados en encontrar factores que promuevan el bienestar en población senescente. Esto es importante, además, por la necesidad que 
existe al interior de la psicología en desarrollar conocimientos sobre la tercera edad (Cicirelli, 1994; APA, 2004), particularmente en los países en vías de desarrollo, en los que la investigación sobre la dimensión funcional del envejecimiento ha sido poco analizada (Eyetsemitan, 2002). Aquí se encontraron datos que permiten comprender las relaciones predictivas que guardan entre sí algunos aspectos de la personalidad en una muestra de adultos mayores, así como la participación de algunas variables socio-demográficas en el esclarecimiento de dichas relaciones.

Específicamente, los resultados apuntan las siguientes tendencias: a) el autoconcepto positivo es mayor en personas que trabajan, tienen un locus de control interno y una menor percepción de características negativas en sí mismo; b) el autoconcepto negativo puede ser explicado por la menor percepción de características personales positivas y por la mayor preocupación ante el fracaso; c) las personas orientadas al éxito son aquellas que al mismo tiempo quieren evitar el fracaso y que también poseen un locus de control interno; d) la preocupación ante el fracaso es mayor entre personas que trabajan, están orientadas al éxito pero también tienen un autoconcepto negativo y una atribución externa sobre la causalidad de los acontecimientos de la vida; e) el locus de control interno es mayor en mujeres, quienes viven con sus nietos, tienen un autoconcepto positivo de sí mismo y están orientados al éxito; f) el locus de control externo tiene una tendencia mayor entre mujeres, quienes tienen un autoconcepto negativo y una combinación de atribución interna y externa acerca del curso de las relaciones interpersonales y; g) la atribución interna-externa del éxito en las relaciones interpersonales es mayor entre las personas con un locus de control externo en la vida.

Estos hallazgos confirman otros resultados encontrados en la literatura. Por ejemplo, Aguilera y Abad (2001) indican que, en la medida en que el adulto mayor considere tener control sobre los acontecimientos de la vida y los cambios asociados a la tercera edad, será más probable que busque alternativas que le permitan adaptarse. Por estar estrechamente vinculado a la autoestima, el autoconcepto positivo es considerado un criterio importante para la valoración geriátrica. De esta forma, locus de control, autoconcepto, junto con la orientación al éxito, pueden ser recursos importantes en la comprensión de la forma en que la personalidad favorece al bienestar y al mantenimiento de la capacidad funcional del adulto mayor. 
Autoconcepto, Locus de Control y Orientación al Éxito: sus Relaciones Predictivas...

Según los estudios realizados por Sandler y Lakey (1982), las personas que poseen características de personalidad como locus de control externo se consideran incapaces de enfrentar los obstáculos con la vida y tienen una tendencia la evitación al fracaso. Con los resultados de esta investigación se observó el poder predictivo del locus de control externo sobre la evitación la fracaso.

Es importante destacar que en las siete ecuaciones de regresión reportadas, las variables de personalidad consiguieron explicar significativamente al criterio en turno. Aún y cuando el bloque de indicadores socio-demográficos no siempre contribuyera en forma significativa a los valores de $R^{2}$, el control de la varianza generado por ellos permitió un entendimiento más claro de las asociaciones entre los distintos aspectos de personalidad analizados.

Los datos obtenidos tienen la dirección prevista por el marco teórico y confirman la interrelación entre aspectos de la personalidad en la tercera edad, sin embargo, la magnitud de los porcentajes de explicación no son altos, pudiendo ser considerados apenas como suficientes para afirmar la existencia de relaciones predictivas entre las variables. Esto puede deberse a la heterogeneidad de la muestra en relación a las variables socio-demográficas que no entraron en las ecuaciones de regresión (ej. edad, ingreso y composición familiar, uso de servicios de salud) así como otras de tipo personal o interpersonal (ej. capacidad funcional, bienestar subjetivo, estrategias de enfrentamiento, apoyo social y dinámica familiar).

Una consideración práctica de los resultados este estudio sugiere el desarrollo de acciones educativas dirigidas a adultos mayores, en que se fomente una percepción positiva de sí mismo, estableciendo de objetivos y metas personales durante la tercera edad, utilizando las capacidades propias y las relaciones interpersonales, al tiempo que se atenúen preocupaciones acerca de posibles fracasos. 
Noriega, J. A. V., Guedea, M. T. D., Álvarez, J. F. L., Albuquerque, F. J. B., \& Seabra, M. (2007). Self concept, locus of control and orientation to exit: their predictive relationships in brasilian northeast adults. Psicologia USP, 18(1), 137-151.

\begin{abstract}
The objective of this work is to analyze predictive relationships between the locus of control, self-concept and the orientation toward achievement in 123 seniors' residents from João Pessoa, Paraiba Brazil. Their ages are between 60 and 93 years olds, the half of them was masculine sex and the other half was feminine sex. The used instruments were: locus of control, self-concept and orientation toward achievement scale, plus a social-demographic questionnaire. Analysis of regression were used, finding diverse predictive relations between personality aspects, confirming the explanatory power of the variables locus of internal and external, autoconcepto positive and negative control and avoidance to failure.
\end{abstract}

Index terms: Internal external locus of control. Self concept. Aged.

Noriega, J. A. V., Guedea, M. T. D., Álvarez, J. F. L., Albuquerque, F. J. B., \& Seabra, M. (2007). Autoconcept, locus de contrôle et orientation dirigée vers la réussite: ses relations predictives chez les adultes qui habite le Nordest brésilien. Psicologia USP, 18(1), 137.151.

Resumé: L'objetif de cet article est d'analiser les relations predictives entre locus de contrôle, autoconcept et orientation dirigées la réussite de 123 agés entre 60 et 93 ans, moitié du sexe féminin et moitié du sexe masculin. Les instruments apliqués sont les suivantes: locus de contrôle, autoconcept et orientation dirigé au réussite validés dans la population qui habite la ville de João Pessoa, et aussi un questionnaire socialdemographique. Ce travail a eté soumis à une analyse de regression hierarchique, dont les résultats ont montré des rélations predictives divers entre les aspects de la personnalité, en entérinant le pouvoir explicatif des variables de locus de contrôle interne et externe, autoconcept positif et negatif et évitement de l'échec.

Descritores: Locus de contrôle. Orientation dirigée vers la réussite. Adultes. 
Autoconcepto, Locus de Control y Orientación al Éxito: sus Relaciones Predictivas...

\section{Referencias}

Aguilera, M., \& Abad, A. (2001). Desarrollo de planes de cuidados en la cartera de servicios de Atención Primaria. Madrid: Instituto Nacional de la Salud.

American Psychological Association. (2004). Guidelines for psychological practice with older adults. American Psychologist, 59(4), 236-260.

Carver, C., \& Scheier, M. (1994). Situacional coping and coping dispositions in a stressful transaction. Journal of Personality and Social Psychology, 66, 184-195.

Cava, M. J., \& Musitu, G. (2000). Bienestar psicosocial en ancianos institucionalizados y no institucionalizados. Multidisciplinar de Gerontología, 10(4), 215-221.

Cicirelli, V. G. (1994). The longest bond: The sibling life cycle. In I. B. Weiner (Org. Série) \& L. L'Abate (Org.Vol.), Wiley series on personality process: Handbook of developmental family psychology and psychopathology (pp. 44-59). New York: Wiley.

Corral, A., \& Ortiz, J. (1990). Los efectos de la institucionalización en la calidad de vida y el autoconcepto en ancianos. La psicología social en México (Vol. III. pp. 23 -28). México: Asociación Mexicana de Psicología Social.

Díaz, L., Galán, S., \& Fernández, G. (1993). Grupo de autocuidado de diabetes mellitus tipo II. Salud Pública de México, 35, 169-176.

Domínguez-Guedea, M. (1999). Autoconcepto, conceptualización de la pareja y depresión en madres: análisis de su relación con la estimulación del desarrollo del niño. Tesis de licenciatura, Universidad de Sonora, Hermosillo.

Domínguez-Guedea, M. (2002). Análise do bem-estar subjetivo em idosos. Dissertação de mestrado, Universidade Federal da Paraíba, João Pessoa.

Emmons, R. (1986). Personal strivings: An approach to personality and subjective wellbeing. Journal of Personality and Social Psychology, 51(5), 1058-1068.

Eyetsemitan, F. (2002). Life-span developmental psychology: Midlife and later years in Western and Non-Western societies. In W. J. Lonner, D. L. Dinnel, S. A. Hayes \& D. N. Satler (Eds.), Online readings in psychology and culture (Unit 12, Chapter 2), Center for Cross-Cultural Research, Western Washington University, Bellingham, Washington USA. http://www.wwu.edu/ culture

Francés, I. (2004). Epidemiología y clínica de la ansiedad en el anciano. In P. Gil y M. Martín. Guía de buena práctica clínica en geriatría. Depresión y ansiedad (pp. 67-79). Madrid: Sociedad Española de Geriatría y Gerontología y Scientific Communication Management.

Gayoso, M. (2004). Epidemiología y clínica. In P. Gil \& M. Martín. Guía de buena práctica clínica en geriatría. Depresión y ansiedad (pp. 13-32). Madrid: Sociedad Española de Geriatría y Gerontología y Scientific Communication Management. 
Herzog, A., Franks, M., Markus, H., \& Holmberg, D. (1998). Activities and well-being in older age: Effects over self concept and educational attainment. Psychology and Aging, 13(2), 179-185.

Hombrados, M., \& García, M. (2002). Control percibido y bienestar subjetivo: un análisis de la literatura gerontológico. Revista Multidisciplinar de Gerontología, 12(2), 90-100.

Horner, M. (1969). Sex differences in achievement motivation and performance in competitive and noncompetitive situations. Dissertation Abstracts International, 30, 4078.

Hudek-Knezevic, J., \& Kardum, I. (1996). A model of coping with conflict between occupational and family roles: Structural analysis. Person Individual Differences, 21(3), 355-372.

Instituto Brasileiro de Geografia e Estatística. (2006). Tendências demográficas. Tabela 17 - Razão de dependência das crianças e dos idosos e índice de envelhecimento, segundo as Grandes Regiões e Unidades da Federação - 1980/2000. Recuperado en 05 de julho de 2006, http://www.ibge.gov.br/home/estatística/populacao/tendenciademográfica/ tabela17.shtm.

Laborín-Álvarez, J. F., \& Páez, R. C. (2000). Bienestar subjetivo y autoconcepto en jóvenes universitarios. In La psicología social en México (Vol. 10, pp. 207-214). Mexico: AMEPSO.

Laborín-Álvarez, J. F., \& Vera, J. A. (2000). Bienestar subjetivo y su relación con el locus de control y el enfrentamiento. In La psicología social en México (Vol. 8, pp. 192-199). Mexico: AMEPSO.

Laborín-Álvarez, J. F., \& Vera, J. A. (2000). Orientación al logro y evitación al éxito en población que habita la región noroeste del desierto de México. Revista Suma, 7(2), 211-230.

López, E. M. (1999). A contribuição da saúde natural à preparação para aposentadoria. In R. Veras (Org.), Terceira idade: alternativa para uma sociedade em transição. Rio de Janeiro : Relume-Dumará.

Martínez, M., \& García, M. (1994). La autopercepción de la salud y el bienestar psicológico como indicador de calidad de vida percibida en la vejez. Revista de Psicología de la Salud, 6, 55-74.

McClelland, D., Atkinson, J., Clark, R., \& Lowell, E. (1961). The achievement motive. New York: Appleton-Century Crofts.

Ministério da Saúde. (2006). Idosos no Brasil. Recuperado en 05 de junho de 2006, http:// dtr2001.saude. gov.br/bvs/exposicoes/idoso/idosob.html

Mora, M., Villalobos, D., Araya, G., \& Ozols, A. (2004). Perspectiva subjetiva de la calidad de vida del adulto mayor, diferencias ligadas al género y a la práctica de la actividad recreativa. Revista MHsalud, 1(1). Disponible en http://www.una.ac.cr/mhsalud/ documents/articulomonicamora_017.pdf 
Autoconcepto, Locus de Control y Orientación al Éxito: sus Relaciones Predictivas...

Moreira, D. A. (2003). Analfabetismo funcional: o mal nosso de cada dia. Multidisciplinar de Gerontología, 10(3),146-151.

Peacock, E., \& Wong, P. (1996). Anticipatory stress: The relation of locus of control, optimism and control appraisals to coping. Journal of Research in Personality, 30(2), 204-222.

Puig, A. (2000). Un instrumento eficaz para prevenir el deterioro cognitivo de los ancianos institucionalizados: el programa de psicoestimulación preventiva (PPP). Revista Multidisciplinar de Gerontología, 10(3), 146-151.

Sandler, I. N., \& Lakey, B. (1982). Locus de control as a stress moderator: The role of control perceptions and social support. American Journal of Psychological Community, 10(1), 65-80.

Sanhueza, M., Castro, M., \& Merino, J. (2005). Adultos mayores funcionales: en nuevo concepto en salud. Ciencia y Enfermería, 11(2), 17-21.

Tabachnick, B., \& Fidell, L. (2001). Using multivariate statistics. New York: Harper Collins.

Teixeira, M. B. (2002). Empoderamento de idosos em grupos direcionados à promoção da saúde. Tesis de Maestría, Fundação Oswaldo Cruz, Escola Nacional de Saúde Pública, Rio de Janeiro, RJ.

Vera-Noriega, J. A., Albuquerque, F. J. B., \& Laborín, J. (1999). Comparación transcultural en rasgos de la personalidad en el desierto latinoamericano: los caso de Sonora-México y Paraíba-Brasil. Proyecto de Investigación. Centro de Investigación en Alimentación y de Desarrollo, Universidad Federal da Paraiba, PB.

Vera-Noriega, J. A., \& Serrano-Quijada, E. (1999). Estructura factorial de un instrumento de auto concepto del adolescente del Estado de Sonora. Revista SESAM, 3(2), 16-19.

Vera-Noriega, J. A., \& Cervantes, N. (2000). Locus de control en una muestra de residentes del Noroeste de México. Revista de Psicología y Salud, 10(2), 237-247.

Vera-Noriega, J. A., Albuquerque, F. J. B., Laborín, J., Morales, A., \& Torres, M. (2002). Autocenceito em uma populacao do Nordeste Brasileiro SCALE Validation of selfconcept in Northeast Brazilians. PSICO: Revista Semestral da Facultade de Psicología da PUCRS, 33(1), 37-51.

Vera-Noriega, J. A., Albuquerque, F. J. B., Laborín, J., Souza, L., \& Coronado, G. (2003). Locus de Controle em uma População do Nordeste Brasileiro. Revista Psicología: Teoria e Pesquisa, 19(3), 211-220.

Recebido em: 18/01/2007

Aceito em: 12/02/2007 